\title{
TUBERCULOSE NO IDOSO: ANÁlISE DO CONCEITO
}

Silvia Helena Figueiredo Vendramini ${ }^{1}$

Tereza Cristina Scatena Villa ${ }^{2}$

Roxana Isabel Cardozo Gonzales ${ }^{3}$

Aline Aparecida Monroe 4

Vendramini SHF, Villa TCS, Cardozo Gonzales RI, Monroe AA. Tuberculose no idoso: análise do conceito. Rev Latino-am Enfermagem 2003 janeiro-fevereiro; 11(1):96-103.

O estudo teve como objetivo definir o conceito de tuberculose no idoso, expresso pela literatura existente na década de 80 e 90. Utilizou-se a análise de conceito, destacando-se os atributos essenciais, eventos antecedentes e conseqüentes do conceito a ser definido. A tuberculose no idoso é expressa como o recrudescimento de infecção longamente inativa. $O$ idoso está mais predisposto à reinfecção, tanto endógena (mais freqüente) como exógena. Dentre os eventos antecedentes, identificouse a moradia nas instituições para idosos como importante fonte de contagio da doença. Os eventos conseqüentes mostraram a necessidade de enfoque diferenciado no diagnóstico e tratamento da doença no idoso, devido às caraterísticas clínicas especificas que o diferenciam dos adultos jovens. Conclui-se a necessidade de realizar novas indagações sobre a análise do conceito desenvolvido, a fim de contribuir na construção de conhecimentos que subsidiem no diagnóstico, tratamento e cuidado do doente idoso.

DESCRITORES: tuberculose, idoso

\section{TUBERCULOSIS IN THE ELDERLY: CONCEPT ANALYSIS}

The goal of this study is to define the concept of tuberculosis in the elderly expressed in the literature covering the 80's and 90's. We have used concept analysis with emphasis on "essential attributes", "preceding events, and "consequential events", which build up the core sense of the concept to be defined. The elderly tuberculosis is expressed as the recrudescence of infection inactivated for a long time. The elderly is predispose to endogenous and exogenous reinfection. Among the most important "preceding" events are the institutional housing as a contact disease source. The "consequential" events are related to the need of a differentiate treatment and diagnostic in elderly due clinical conditions. We arrive at the conclusion that new studies about the concept analysis need to be developed in order to contribute to the knowledge about the diagnosis, treatment and care to elderly patients.

\section{DESCRIPTORS: tuberculosis, elderly}

\section{TUBERCULOSIS EN EL ANCIANO: ANÁLISIS DE CONCEPTO}

El objetivo del estudio fue definir el concepto de "tuberculosis en el anciano" existente en la literatura científica de la década de 80 y 90. Se utilizó el análisis de concepto, destacándose los atributos esenciales, eventos antecedentes y consecuentes del concepto. La tuberculosis en el anciano se expresa como el recrudecimiento de infección largamente inactiva. El anciano presenta mayor predisposición para reinfección endógena (más frecuente) y exógena. Entre los eventos antecedentes, se identificaron las instituciones para ancianos como importante fuente de contagio de la enfermedad. Los eventos consecuentes, mostraron la necesidad de enfocar de forma diferenciada el diagnóstico y tratamiento de la enfermedad por la presencia de características propias de la tuberculosis en este segmento de la población. En conclusión, se considera importante realizar nuevas investigaciones respecto al concepto desarrollado con la finalidad de aportar para el diagnostico, tratamiento y cuidado del enfermo anciano.

\section{DESCRIPTORES: tuberculosis, anciano}

\footnotetext{
${ }^{1}$ Enfermeira, Doutoranda em Saúde Pública, e-mail: descop@famerp.br; ${ }^{2}$ Professor Doutor, e-mail: tite@eerp.usp.br; ${ }^{3}$ Enfermeira, Doutoranda em Saúde Pública, e-mail: roxana_cardozo@hotmail.com; ${ }^{4}$ Enfermeira, Mestranda em Enfermagem em Saúde Pública, e-mail: Aline_monroe@zipmail.com.br. Escola de Enfermagem de Ribeirão Preto da Universidade de São Paulo, Centro Colaborador da OMS para o desenvolvimento da pesquisa em enfermagem
} 
INTRODUÇÃO

Tuberculose no idoso

A tuberculose é uma doença infecciosa crônica que, na ausência de tratamento eficaz, evolui para a doença ativa, de forma consuntiva, sobrevindo, como última conseqüência, a morte. Às vezes, a doença no adulto é o resultado de um novo inóculo de bacilos em uma pessoa já sensibilizada por uma infecção prévia (reinfecção exógena) $)^{(1)}$.

O diagnóstico da tuberculose deve ser sempre precedido pela anamnese, exame físico e a radiografia de tórax que pode auxiliar no diagnóstico da maioria dos casos. A bacteriologia ocupa um papel fundamental, permitindo, por meio do conhecimento dos vários aspectos da biologia do bacilo, a sua correta identificação(2).

O teste tuberculínico, realizado com PPD, tem importância, sobretudo na detecção da infecção pelo bacilo e não na determinação da atividade da doença ${ }^{(3)}$. A prova tuberculínica positiva, isoladamente, indica apenas infecção e não necessariamente tuberculose doença.

A terapêutica utilizada combina várias drogas, uma vez que o bacilo causador apresenta, rotineiramente, mutações $^{(3)}$.

A população mundial está envelhecendo, e projeções apontam que, de 1975 ao ano 2025, a população idosa terá crescido $224 \%$, enquanto a população geral crescerá $102 \%$, cabendo aos países em desenvolvimento abrigarem 2/3 dessa população com 60 anos e mais ${ }^{(4)}$.

A literatura científica, nacional e internacional, produzida sobre o assunto, considera idoso o indivíduo que está na faixa etária de 60 anos e mais. Nesse sentido, neste estudo, utilizam-se os termos: velho, idoso, terceira idade, dentre outras expressões, para designar esse grupo da população, sem a preocupação de se ajustar uma expressão adequada e única ${ }^{(5)}$.

O Brasil passa por uma transição demográfica, cuja conseqüência tem sido o aumento do segmento da população de idosos, apresentando caraterísticas que os diferenciam do resto da população ${ }^{(6)}$. O processo de envelhecimento é dinâmico, apresentando modificações tanto morfológicas como funcionais, bioquímicas e psicológicas, que determinam a progressiva perda da capacidade de adaptação do indivíduo ao meio ambiente, ocasionando maior vulnerabilidade e maior incidência de processos patológicos que terminam por levá-lo à morte ${ }^{(7)}$. Existe uma maior vulnerabilidade dos velhos para adoecer $^{(8)}$.

Concomitante ao aumento desta população vulnerável, vem-se observando o aumento do número de casos de tuberculose.

Nos países desenvolvidos, essa doença acomete, mais freqüentemente, as pessoas idosas, assim como as minorias étnicas e imigrantes estrangeiros. Devido ao aumento da incidência da doença na população idosa, desde meados de 1980, houve um interesse por investigar a doença em pessoas acima de 65 anos $^{(9)}$.

A proporção de casos de tuberculose em idosos no Brasil, entre 1986 e 1996, subiu de 10,5 por cento para 12 por cento, e a mediana de idade de 38 para 41 anos $^{(10)}$. O menor declínio do coeficiente de incidência ocorreu nas faixas de 30-49 anos e 60 anos e mais. Entre 1980 e 1996, a mediana de idade dos óbitos subiu de 53 para 55 anos. O declínio generalizado dos coeficientes de mortalidade observado entre 1986 e 1991 tornou-se menos expressivo, nas faixas etárias de 30 anos e mais, entre 1991 e 1996. Houve correlação direta entre idade e mortalidade. A maior proporção de casos sem confirmação bacteriológica ocorreu em idosos ${ }^{(11)}$.

A incidência de tuberculose no Brasil começa a ser deslocada para a faixa etária correspondente aos idosos. Para tal fato, contribuem, por um lado, a eficácia da vacinação $B C G$ e a redução do risco de infecção na comunidade; por outro, o crescimento da população de adultos e idosos. O diagnóstico difícil nessa faixa etária determina elevada mortalidade. Nos próximos 50 anos deverá ocorrer redução progressiva dos casos associados à AIDS em adultos, e expressivo aumento dos casos de reativação em idosos, cuja população, no Brasil, saltará de 5 por cento para 14 por cento. A proporção de idosos é estimativa dos demógrafos ${ }^{(12)}$.

Face ao exposto, o interesse temático desta pesquisa justifica-se por considerar que a tuberculose no idoso é um problema de saúde emergente e complexo, devido à própria patologia e à presença de outras caraterísticas ou fatores que favorecem a gravidade da doença.

\section{OBJETIVO}

Definir o conceito de tuberculose no idoso, expresso pela literatura geral, nas décadas de 80 e 90 , por meio dos atributos essenciais, antecedentes e conseqüentes do conceito. 
REFERENCIAL TEÓRICO METODOLÓGICO

O que se entende por conceito?

A palavra conceito ${ }^{(13)}$ deriva do latim conceptus e significa "representação de um objeto pelo pensamento, por meio de suas características gerais. A ação de formular uma idéia por meio de palavras; definição e caracterização".

Nesse sentido, o desenvolvimento de conceito pode ser entendido como um processo, uma construção mental resultante das observações e experiências em torno dos fenômenos.

O conceito é entendido como representação cognitiva, abstrata, de realidade perceptível, formada por experiências diretas ou indiretas e que pretende corresponder às características fundamentais do fenômeno observado, inserido, portanto, em um contexto, de modo que o significado e aplicação sejam possíveis, podendo contribuir para o avanço do conhecimento ${ }^{(14)}$.

As maneiras de desenvolver conceitos em qualquer área, sistematicamente, perpassam três estágios: "a análise do conceito", "a síntese do conceito", e a "derivação de conceito", sendo que a sua escolha depende do contexto ${ }^{(15)}$.

Quanto mais claros forem os conceitos, ou seja, a expressão de seus "atributos essenciais", maior será o entendimento entre aqueles que o utilizam. Esses atributos são compreendidos como características que aparecem com freqüência, quando um conceito é descrito ${ }^{(15)}$.

A metodologia expressa oito fases que não exigem execução mecânica. O fundamental é destacar a intenção e a dimensão na "análise do conceito", estabelecendo relações entre as fases que se encontram apresentadas abaixo:

- identificar o conceito de interesse e expressões associadas;

- identificar e selecionar campo apropriado para coleta de dados;

- coleta de dados, reconhecendo os atributos do conceito e também os termos substitutos, relacionados, antecedentes e conseqüentes;

- analisar os dados e identificar características dos conceitos;

- identificar antecedentes, conseqüentes e termos substitutos;

- identificar caso-modelo do conceito, se apropriado;

- identificar hipóteses e implicações para outros estudos.
Esse modelo de análise foi utilizado no estudo, com algumas variantes, pois existem alguns aspectos que não foram explorados, tais como: termos substitutos, relacionados, caso-modelo, hipótese e implicações para outros estudos.

Campo de estudo - literatura e coleta de dados

Realizou-se pesquisa documental, e a construção de seus passos direcionou-se à identificação dos autores na literatura existente, os quais publicaram estudos sobre tuberculose no idoso.

O campo de coleta de dados foi na Biblioteca Central da Universidade de São Paulo, Campus Ribeirão Preto.

Inicialmente, realizou-se uma consulta preliminar baseada em artigo publicado em periódico de língua portuguesa, que serviu como referência para a busca manual de outros artigos, nos periódicos da Biblioteca Central da USP-Ribeirão Preto.

Posteriormente, foi realizada a busca eletrônica no Banco de dados da "Literatura Latina Americana e Caribe em Ciências da Saúde" (LILACS) e MEDLars on LINE (MEDLINE).

Realizou-se levantamento bibliográfico, com corte histórico relativo aos trabalhos publicados na década de 80 e 90, utilizando-se as seguinte palavras-chave: tuberculose e idoso e tuberculosis and aged.

Foram estabelecidos critérios de inclusão para delimitar o corpo documental de análise, sendo necessária a palavra-chave no título e resumo do artigo. Contemplouse, então, um total de 22 artigos; 06 foram encontrados em outras referências, 12 na MED LINE e 04 no LILACS.

Após esses passos, procedeu-se à aquisição dos artigos selecionados, via COMUT - Biblioteca Central. Dos quatro artigos selecionados pela LILACS, foram adquiridos três artigos, sendo que um desses, não foi encontrado na rede brasileira.

Foram excluídos os artigos selecionados pela MED LINE, devido ao fato de a maioria dos artigos estar publicada em outras línguas não dominadas, principalmente japonesa e alemã. Desse modo, os artigos selecionados e utilizados para a análise do conceito de tuberculose no idoso, após os recortes necessários, totalizaram 9 artigos.

A partir desse conjunto de documentos, tornouse possível definir o corpo de análise. Submeteu-se cada 
documento à leitura exploratória, para apreciação do título, conteúdo e para verificar se os mesmos atendiam aos critérios: abordagem do tema e desenvolvimento do conceito de tuberculose no idoso.

Esse momento de leitura permitiu refinar a sensibilidade no reconhecimento do conceito atribuído pelos autores. Identificaram-se como recorte: frases, temas, palavras/termos, expressões e, quando possível, parágrafos, registrando-os em folha à parte, para melhor organizar a "análise do conceito".

\section{CONSTRUINDO O CONCEITO DE INTERESSE - "TUBERCULOSE NO IDOSO"}

Atributos do conceito em estudo

Para a identificação dos atributos essenciais que discutem a tuberculose no idoso, utilizaram-se as questões: Como o autor define o conceito? Quais as caraterísticas/atributos apontados por ele? Que idéias o autor discute sobre a tuberculose no idoso ${ }^{(16)}$.

Consideram-se "atributos" as palavras e/ou expressões utilizadas com freqüência pelos autores e apresentadas como afirmação dos conceitos elaborados, sendo esses "atributos" tidos como essenciais ${ }^{(17-18)}$.

Dentre os "atributos" identificados na análise dos nove artigos, alguns foram mais citados pelos autores, conforme ilustra a Figura 1.

\begin{tabular}{|l|c|}
\hline ATRIBUTOS ESSENCIAIS & № DE AUTORES \\
\hline Recrudescimento & 6 \\
\hline Apresentação atípica & 5 \\
\hline Reinfecção endógeno/exógena & 4 \\
\hline Quimioterapia antituberculosa & 3 \\
\hline Transmissível & 2 \\
\hline Duração do tratamento imprevisível & 1 \\
\hline
\end{tabular}

Figura 1 - Distribuição dos "atributos essenciais" da "tuberculose no idoso" segundo o número de autores analisados nos anos de 1980 a 1999

O "atributo" denominado recrudescimento é entendido como tornar-se mais intenso, agravar-se, aumentar, exacerbar-se, recrescer. Atípica significa que se afasta do normal, do caraterístico. Reinfecção é compreendida como nova infecção, com os germes causadores de uma infecção anterior. Quimioterapia significa o tratamento por meio de agentes químicos que interferem sobre uma determinada doença. Infecção é entendida como a contaminação, penetração, desenvolvimento e multiplicação de seres inferiores no organismo do hospedeiro, de que podem resultar, para ele, conseqüências variadas, habitualmente nocivas, em grau maior ou menor. Transmissível significa comunicar por contagio; propagar, passar de uma pessoa para outra. Imprevisível é entendido como algo que não pode ser antecipado, calculado, prognosticar ou predizer ${ }^{(13)}$.

Evidenciam-se esses "atributos" referidos nos conceitos expressos pelos autores mencionados abaixo:

"A doença é o resultado de recrudescência de infecção longamente inativa, pois as pessoas idosas são as únicas que experimentaram a mais extensa exposição nas suas vidas"(19).

"A tuberculose pulmonar dos idosos na maioria das situações se mostra de forma atípica"(20).

"Os idosos estão mais predispostos à reinfecção, tanto endógena como exógena ${ }^{(21)}$, no entanto, a reinfecção endógena é a mais freqüente, especialmente nos meios sociais desenvolvidos"(20).

"O tratamento da tuberculose no idoso não foge às leis gerais da terapêutica antituberculosa"(20).

"A doença é mais facilmente transmitida em ambientes fechados e com exposição prolongada"(21).

"O tratamento é imprevisível, especialmente nas formas hematogênicas extensas, dificilmente seu prazo é inferior a doze meses e assim mesmo deve-se contar com reativações futuras, após cessação do tratamento"(20).

Os idosos de hoje em dia viveram a infância e a juventude em meados do século XX, numa época de alta prevalência da doença e em que o tratamento efetivo era inexistente, pois a comprovação da eficácia dos quimioterápicos no tratamento da tuberculose foi alcançada somente ao longo das décadas de 50 e 60 . Nesse período, anterior à descoberta do tratamento, foram expostos aos bacilos, albergando, dessa maneira, a bactéria quiescente da infecção ou das lesões fibróticas decorrentes da "cura" espontânea ${ }^{(22)}$.

Eles seriam os grandes reservatórios da bactéria na atualidade, principalmente em países desenvolvidos como os Estados Unidos, onde o coeficiente/100.000 habitantes, em 1996, era de $7,9^{(23)}$. Por causa disso, $95 \%$ dos casos dos idosos têm a tuberculose por reativação de foco endógeno ${ }^{(24)}$

$\mathrm{Na}$ análise dos artigos, observou-se que os autores relatam que a representação clínica da doença 
pulmonar nas pessoas idosas não difere significativamente, porém eles sugerem levar em consideração as caraterísticas clínicas, radiológicas e laboratoriais do idoso, no momento do diagnostico ${ }^{(25)}$, os meios de adquirir a doença também devem ser diferentes nas pessoas velhas.

Em relação ao tratamento da doença no idoso, identificou-se, por meio da análise, que não difere do adulto jovem.

Em relação à transmissão da doença ${ }^{(22)}$, ocorre mais facilmente em ambientes fechados ou instituições para idosos. Esses lugares constituem-se nas fontes mais férteis de tuberculose e de sua transmissão.

Considera-se que a análise do conceito de "Tuberculose no idoso", por meio dos atributos essenciais, é importante para favorecer a compreensão mais ampliada desse conceito que se apresenta de forma constante e progressiva na população idosa.

Antecedentes do conceito

Entende-se por "antecedentes" aquelas situações, eventos ou fenômenos que precedem ao "conceito de interesse". Auxiliam a compreensão do contexto social, no qual o conceito é geralmente usado, como também favorece o refinamento do mesmo.

No presente estudo, identificaram-se os eventos "antecedentes" por meio das respostas à pergunta: Que eventos contribuem para a iminência do conceito de tuberculose no idoso?

Os artigos examinados revelaram eventos "antecedentes" que emergem com maior freqüência, ilustrados na Figura 2.

\begin{tabular}{|l|c|}
\hline ANTECEDENTES & № DE AUTORES \\
\hline Moradia em instituição para idosos & 7 \\
\hline Aumento da incidência & 6 \\
\hline Idade avançada & 5 \\
\hline Imunodeprimidos & 4 \\
\hline Má alimentação & 3 \\
\hline Doenças associadas & 1 \\
\hline Alcoolismo & 1 \\
\hline Tabagismo & 1 \\
\hline Drogas & 1 \\
\hline
\end{tabular}

Figura 2 - Distribuição dos eventos antecedentes para o conceito de "tuberculose no idoso", segundo o número de autores analisados nos anos de 1980 a 1999

Identifica-se a instituição para idosos como o evento antecedentes mais citado pelos autores para o surgimento do conceito de "tuberculose no idoso".

Os antecedentes idade avançada, imunodeprimidos, má alimentação, as doenças associadas, alcoolismo, tabagismo e drogas também são apontadas dentre outros como fatores responsáveis por uma maior morbidade e letalidade da tuberculose no idoso.

Os eventos "antecedentes" na Figura 2 são identificados nas citações dos autores, conforme mostrados abaixo:

"Tem-se observado que as instituições para idosos são lugares de alto risco de transmissão da tuberculose, provavelmente por serem ambientes fechados"(26).

"Desde meados de 1980, há interesse pelo aumento da incidência de tuberculose pulmonar nas pessoas acima de 65 anos"(25).

"Os idosos estão mais predispostos a contrair a doença, pela depressão das defesas orgânicas na idade avançada e condições imunossupressoras associadas"(21).

"Dentre os fatores que favorecem a aparição da tuberculose no idoso devem-se citar doenças associadas, alcoolismo, tabagismo, má alimentação"(25-27).

No envelhecimento, a imunidade celular do indivíduo, considerada como a maior responsável pelo combate à infecção tuberculosa, diminui. Em conseqüência, suas funções de proteção do organismo encontram-se afetadas, favorecendo a presença de doenças infecciosas ${ }^{(28)}$.

As doenças associadas constituem-se um fator importante verificado nos idosos da América Latina e caraterizado pela situação econômica e falta de acesso aos serviços de saúde ${ }^{(29)}$.

Na Inglaterra, país desenvolvido, observou-se que idosos residentes em áreas urbanas e vivendo em condições de privação, tiveram duas vezes mais notificações de tuberculose, realçando-se a importância da pobreza e da privação como determinantes sociais negativos para o acometimento dessa doença ${ }^{(30)}$.

\section{Conseqüentes do conceito}

Os conseqüentes do conceito referem-se a eventos ou situações resultantes da "tuberculose no idoso", evidenciáveis na aplicação efetiva do conceito. Para identificá-los, utilizou-se a seguinte questão: $O$ que acontece depois que se constatou a tuberculose no idoso? 
Os eventos "conseqüentes" são úteis para fornecer novas idéias às pesquisas, permitindo investigação mais ampla de todas as facetas do conceito e favorecendo uma perspectiva maior do estudo ${ }^{(15)}$.

Nos artigos examinados a respeito do conceito de interesse, identificaram-se eventos "conseqüentes" discutidos na análise dos atributos e antecedentes da tuberculose no idoso.

Indicações de novas necessidades vêm ilustradas na Figura 3.

\begin{tabular}{|l|c|}
\hline CONSEQÜENTES & № DE AUTORES \\
\hline Necessidade de enfoque diferenciado & 5 \\
\hline Propagação nosocomial & 4 \\
\hline Efeitos adversos freqüentes & 2 \\
\hline Mortalidade & 2 \\
\hline Prognóstico severo & 1 \\
\hline Necessidade de repouso & 1 \\
\hline
\end{tabular}

Figura 3 - Distribuição dos eventos conseqüentes do conceito de "tuberculose no idoso", segundo número de autores analisados nos anos 1980 a 1999

Analisando os eventos conseqüentes da tuberculose no idoso, destacou-se a necessidade de enfoque diferenciado no diagnóstico e tratamento do doente idoso de tuberculose, devido a algumas caraterísticas clínicas diferenciadas da doença nessa população, assim como por apresentar outros fatores que tornam esses casos mais freqüentes:

- Em geral, os sintomas comuns na tuberculose, tais como tosse, perda de peso, fraqueza e dispnéia, além de sutis, são, freqüentemente, dados como sendo de outras doenças, como bronquite crônica do fumante e enfisema; - Nos idosos, são pouco freqüentes os sintomas sugestivos da doença: sudorese noturna e hemoptise;

- A hipertermia costuma ser baixa e pouco aparente a não ser quando medida ${ }^{(31)}$.

Dentre os outros eventos conseqüentes, identificaram-se os efeitos adversos freqüentes por causa do tratamento; mortalidade, prognóstico severo e necessidade de repouso.

Os eventos "conseqüentes" mais freqüentes expressados pelos autores são apresentados abaixo:

“...dever-se-ão reforçar as diferenças entre a forma atípica da doença no idoso e o modelo clássico visto em pacientes jovens; além de dar ênfase às dificuldade de diagnóstico nos indivíduos idosos”(25).
"A doença pode alcançar proporções epidêmicas em ambientes fechados como as instituições e lares para idosos"(25).

"As drogas antituberculose freqüentemente podem causar reações adversas no idoso"(27).

"A tuberculose no idoso é de prognóstico severo, e os índices de mortalidade continuam elevados"(27).

Nos artigos, os autores consideram como causa importante para seus estudos o fato de a tuberculose no idoso ocorrer, principalmente, em ambientes fechados, como instituições e lares para essa população, podendo alcançar proporções epidêmicas entre as idades susceptíveis.

Em relação aos efeitos adversos ocasionados pela droga, isso acontece, principalmente, devido ao fato de o idoso apresentar alterações. As doses de medicamentos a serem administradas devem ser muito bem estabelecidas em relação ao peso, particularmente a estreptomicina e outros aminoglicosídeos, para os quais se recomenda a metade da dose preconizada para o adulto jovem $^{(21)}$.

\section{CONSIDERAÇÕES FINAIS}

No percurso da análise, reconheceu-se a natureza dinâmica do conceito da tuberculose no idoso. O conceito revelou, como uns dos principais atributos essenciais mais citados pelos autores, o recrudescimento de infecção longamente inativas. Atribui-se o envelhecimento (diminuição da imunidade celular), doenças associadas, alcoolismo, dentre outros, como fatores que favorecem a presença da tuberculose no idoso.

A apresentação clínica da doença é um aspecto importante a ser destacado na tuberculose no idoso, devido a algumas caraterísticas que o diferenciam dos outros segmentos da população. Essas caraterísticas adquirem vital importância no diagnostico e tratamento da doença.

$\mathrm{Na}$ análise dos antecedentes do conceito, um dos eventos mais citados foi a moradia nas instituições para idosos. Esses ambientes constituem grandes fontes de transmissão da doença. Nesse sentido, considera-se relevante alertar aos trabalhadores da saúde e/ou funcionários destas instituições a ficarem atentos na identificação de sinais e sintomas da doença, principalmente no idoso que apresenta outros problemas 
de saúde (imunodepressão, doenças associadas, alcoolismo, tabagismo, drogas) a fim de favorecer o diagnóstico precoce da tuberculose.

Dentre os eventos conseqüentes mais citados, destaca-se a necessidade de um enfoque diferenciado da doença no idoso. Embora os autores refiram que não existe uma diferença significativa da tuberculose no idoso, comparada com a do adulto jovem, e ressaltadas as caraterísticas especificas presentes no idoso, por tal motivo, torna-se importante considerar essas caraterísticas não comuns no momento do diagnóstico e tratamento, a fim diminuir os danos no idoso e o risco de morte.

Diante do exposto, considera-se que o estudo permitiu apresentar alguns aspectos que necessitam de

\section{REFERÊNCIAS BIBLIOGRÁFICAS}

1. Campos ML, Cipriano ZM, Stamm AM de F, Tratsk KS. Tuberculose. Rev Bras Med 2000 jul; 57(6):505.

2. Ministério da Saúde (BR). Fundação Nacional de Saúde. Distribuição de casos novos notificados por forma clínica e coeficiente de incidência de positivos e todas as formas por grupo etário. [on line] Disponível em: URL: http//www. fns.gov.br/ações/doenças/tub/tuber $9 \mathrm{htm}$ [acessado em 16 de jun de1998].

3. Gerhart G Filho. Controle da tuberculose. Uma proposta de integração Ensino-Serviço. 3a ed Rio de Janeiro: Ministério da Saúde; 1992.

4. Fundação Seade (SP). São Paulo em Dados. 2001 [on line] Disponível em: URL: http://www.seade.gov.br

5. Beavoir S. A velhice. Rio de Janeiro: Nova Fronteira; 1990. 6. Baldessin A. O idoso: viver e morrer com dignidade. In: Papaléo M Neto. Manual de Gerontologia. São Paulo: Atheneu; 1996. p. 491-8.

7. Carvalho ET Filho, Alencar YMG de. Teorias do envelhecimento. In: Carvalho ET Filho, Papaléo M Neto. Manual de Geriatria: fundamentos, clínica e terapêutica. São Paulo: Atheneu; 1994. p.1-8

8. Rodrigues, RAP. Mulheres em mudanças no processo de vida e envelhecer. Ribeirão Preto. [Tese]. Ribeirão Preto (SP): Escola de Enfermagem/USP; 1997.

9. Powell KE, Farer LS. The rising age of the tuberculosis patient: a sign of success and failure. J Infect Dis 1980; (142):946-8.

10. Ministério da Saúde (BR). Fundação Nacional de Saúde: Centro Nacional de Epidemiologia. Programa de Controle da Tuberculose. Disponível em http://www. funasa.gov.br. [Acessado em 16 de junho de 2001].

11. Ministério da Saúde: Secretaria executiva. Datasus. Informações em Saúde: mortalidade. [on line]. Disponível em www. datasus.gov.Br. [Acessado em 28 de junho de 2001].

12. Chaimowicz $F$ Age transition of tuberculosis incidence and mortality in Brazil. Rev Saúde Publica; 2001; 35(1):81-7. 13. Ferreira $A B$ de $H$. Novo Aurélio Século $X X I$ : o dicionário da língua portuguesa. $3^{\underline{a}}$ ed. Rio de Janeiro (RJ): Nova Fronteira; 1999. revisão, devendo ser levados em conta no intuito de orientar o cuidado à saúde nessa população, cada vez maior, principalmente quando se considera a confluência de vários fatores negativos que favorecem a presença da doença de maneira mais cruel.

Espera-se que novas indagações e críticas sejam suscitadas pela análise do conceito, contribuindo, assim, para a continuidade da construção do conhecimento científico.

No Brasil, são poucos os estudos sobre o assunto, sendo pertinente a recomendação de pesquisas em nível nacional, inclusive pela enfermagem, pois não foi encontrado nenhum artigo dessa área sobre tuberculose no idoso.

14. Srour RH. Classes, regimes e ideologias. São Paulo (SP): Ática; 1990.

15. Walker L Avant KC. Strategies for theory constrution in nursing. California: Apleton \& Lange; 1988.

16. Rodgers BL. Concept, analysis and the development of nursing knowledge: the evolutionary cycly. Adv Nurs 1989; 14:330-4.

17. Rodgers BL Knafl DA. Concept development in nursing: foundations, techniques, and applications. Philadelphia: WB. Sauders; 1993.

18. Morse JPM. Exploring the theoretical basis of nursing using advanced techniques of concept analysis. Adv Nurs Sci 1995; 17(3):31-46.

19. Stead WW Lofgren J P. Medical perspective:does the risk of tuberculosis increase in old age? J Infect Dis 1983; 147: 951-5.

20. Paula A de. Tuberculose e velhice. Arq Bras Med 1988 mar/abr; 62(2):113-7.

21. Duarte $S$ Nascimento $V$ do; Sgaib NM Tuberculose no idoso. J Pneumol 1993; 19(2):96-8.

22. Davidson PT. Tuberculosis: new views as an old disease. N Engl J Med 1985; 312:1514-5.

23. Ministério da Saúde (BR). Fundação Nacional de Saúde. Centro Nacional de Pneumologia Sanitária. Centro de Referência Hélio Fraga. Plano Nacional de Controle da Tuberculose. Brasília. 1999.

24. Dutt AK Stead WW. Tuberculosis in the elderly. Med Clin North Am 1993; 77:1353-68.

25. Morris CDW. Pulmonary tuberculosis in the elderly: a different disease? Thorax 1990; (45):912-3.

26. Villareal Velarde $\mathrm{H}$, Vargas $\mathrm{MH}$, Torres Cruz A, Urueta Robledo J, Pérez Guzmán C. et al. Tuberculosis pleuropulmonar en el anciano: estudio comparativo com otras edades. Rev Inst Nac Enfermedades Respir 1998 abr/jun; 11(2):111-6.

27. Pilheu JA. Tuberculosis en la edad avanzada. Rev Argentina Tuberc Enfermedades Pulm Salud Publica 1985 jan/mar; 46(1):55-63.

28. Chaimowicz F. Tuberculose em idoso. In: Rocha MOC, Pedroso ERP, Santos AGR. Infectologia geriátrica. São Paulo: Fundo Editorial Byk; 1997. p. 193-220. 
29. Lituak J. El envejecimiento de la poblacion: un desafio que va más allá del ãno 2000. Bol Of Sanit Panam 1990; 109(1):1-5.

30. Kearney MT, Wanklin PO, Goldman JM, Pearson SB, Teale C. Urban deprivation and tuberculosis in the elderly. Resp Med 1994; 88:703-4.

31. Mishima EO. Tuberculose no Idoso: Estudo de São Paulo. [Dissertação]. São Paulo: Faculdade de Saúde Pública/USP; 2000. 